\title{
The Numerical Solution of Fractional Bratu-Type Differential Equations
}

\author{
Duygu Dönmez Demir ${ }^{1 *}$ and Aylin Zeybek ${ }^{2}$ \\ *Department of Mathematics, Manisa Celal Bayar University, Manisa, Turkey
}

\begin{abstract}
This paper introduce the differential transform method (DTM) to solve the fractional Bratu-type differential equation modeling a combustion in numerical slab. For the definition of fractional derivative, the Caputo sense is used. The result corresponds to the exact solution when the obtained solution is constructed as power series for some values of fractional order. Finally, some examples are presented to indicate the efficiency of applied method. Comparison of the results obtained by DTM with those obtained by other methods is given.
\end{abstract}

Keywords: Fractional Bratu-type differential equation; DTM; Caputo sense fractional derivative.

\section{Introduction}

The mathematical model of many events in nature may be obtained by fractional order differential equations. Fractional differential equations have various applications in many fields such as physics, engineering, fluid and statistical mechanics, biosciences and electrochemistry. Since the exact solutions of fractional differential equations do not exist, many researchers focus on approximate solutions of functional equations of fractional order in particular. There are several numerical methods given for differential equations of integer order which extended to fractional order differential equations. These methods are known as differential transform method (DTM), variational iteration method (VIM), Adomian decomposition method (ADM), homotopy perturbation method (HPM) and etc. [1]. This study focuses on the solution of Bratu-type fractional differential equation [2]

$$
\begin{gathered}
D^{\alpha} y(x)+\lambda \exp [y(x)]=0,1<\alpha \leq 2,0<x<1 \\
y(0)=b_{1}, \quad y^{\prime}(0)=b_{2}
\end{gathered}
$$


where $D^{\alpha}$ represents the derivative operator of fractional order $\alpha, y(x)$ is unknown function on the interval $[0,1]$ and $b_{1}, b_{2}$ and $\lambda$ are the constants given. The numerical solutions of the mentioned problem have been investigated by ADM [1], HPM [3], VIM [4], RKM [2] before.

We consider DTM in this study. The DTM is an iterative method based on Taylor's series expansion [5]. This technique has been developed by Elsaid [6] as fractional differential transform method (FDTM) and by Odibat [7] et al. as generalized differential transform method (GDTM) for fractional differential equations. In this study, we used FDTM. For exponential nonlinearity, its Adomian polynomials are considered, then, the differential transform of the dependent variable components can be obtained. The numerical results for the different examples having exponential nonlinearity are compared with exact solutions and RKM solutions. It is concluded that the present method gives good results for Bratu-type fractional differential equations.

\section{Basic Concepts and Definitions}

In this section, we introduce necessary definitions related to fractional calculus which is needed in our study.

\subsection{Definition [6].}

A real function $f(x), x>0$ is said to be in the space $C_{\eta}, \eta \in \mathrm{i}$, if there exists a real number $p>\eta$, such that $f(x)=x^{p} g(x)$, where $g(x) \in C(0, \infty)$ and it is said to be in the space $C_{\eta}^{n}$ if $f^{(n)} \in C_{\eta}, n \in ¥$.

\subsection{Definition [8].}

In the fractional calculus, the Caputo's fractional derivative is the definition which the initial conditions give most appropriately to the physical conditions. The Caputo's fractional derivative is defined by

$$
{ }_{a}^{C} D_{x}^{\alpha} f(x)=\frac{1}{\Gamma(n-\alpha)} \int_{a}^{x} \frac{f^{(n)}(\xi)}{(x-\xi)^{\alpha+1-n}} d \xi ; n-1<\alpha<n .
$$

The Caputo's definition has an advantage since it has the initial conditions in same form with those of the equations of integer order.

\subsection{Definition [8].}

For $n-1 \leq \alpha<n, n \in Z^{+}, x>a$, the definition of the fractional derivative in the RiemannLiouville sense is 


$$
{ }_{a}^{R L} D_{x}^{\alpha} f(x)=\frac{1}{\Gamma(n-\alpha)} \frac{d^{n}}{d x^{n}} \int_{a}^{x} \frac{f(\xi)}{(x-\xi)^{\alpha+1-n}} d \xi .
$$

\subsection{Definition [6].}

The fractional integral of order $\alpha \geq 0$ in the Riemann Liouville sense of a function $f(x) \in C_{\eta}, \eta \geq-1$ is defined as

$$
I_{a}^{\alpha} f(x)=\frac{1}{\Gamma(\alpha)} \int_{a}^{x}(x-\xi)^{\alpha-1} f(\xi) d \xi ; \quad \alpha>0, x>0
$$

Also, the Caputo fractional derivative [6] can be given by

$$
\begin{aligned}
& { }^{C} D^{\alpha} f(x)=I^{n-\alpha} \frac{d^{n}}{d x^{n}} f(x) ; n-1<\alpha<n \\
& { }^{C} D^{\alpha} f(x)=\frac{d^{n}}{d x^{n}} f(x) ; \alpha=n
\end{aligned}
$$

\section{Fractional Differential Transform Method}

DTM is considered for the approximate solutions of many differential equations appearing in the fields such as the engineering and sciences. This method produces result without any linearization, perturbation and restriction. DTM is based on Taylor's series expansion constructed in polynomial form. This method has a solution of polynomial series obtained as iterative. Recently, the mentioned technique is effectively used for linear or nonlinear fractional differential equations. We consider analytic function

$$
y(x)=\sum_{k=0}^{\infty} Y(k)\left(x-x_{0}\right)^{\frac{k}{\theta}}
$$

where $\theta$ denotes the order of fraction to be choosed and $Y(k)$ is the $k$ th fractional differential transform of $y(x)$. Applying the initial conditions by integer-order derivatives for practical applications, then the transformation of initial conditions is obtained as

$$
Y(k)= \begin{cases}\frac{1}{(k / \theta) !}\left[\frac{\partial^{k / \theta}}{\partial x^{k / \theta}} y(x)\right]_{x=x_{0}}, \frac{k}{\theta} \in \phi^{+} \\ 0 \quad, \frac{k}{\theta} \notin \phi^{+}\end{cases}
$$

where $k=0,1, \mathrm{~K},(\alpha \theta-1)$ and $\alpha$ is order of the fractional differential equation [6]. Here, $\theta$ should be selected where $\alpha \theta$ is a positive integer. Besides, the fractional differential transform method has some fundamental properties in the following; 
We assume that $y(x), z(x)$ and $r(x)$ are the functions of variable $x$ and $Y(k), Z(k)$ and $R(k)$ are their differential transforms with order of fraction $\theta$. Thus, the properties are given as;

- If $y(x)=z(x) \pm r(x)$, then $Y(k)=Z(k) \pm R(k)$.

- If $y(x)=c z(x)$, then $Y(k)=c Z(k)$ for $c$ is a constant.

- If $y(x)=D_{x}^{\alpha} z(x)$, then $Y(k)=\frac{\Gamma\left(\alpha+1+\frac{k}{\theta}\right)}{\Gamma\left(1+\frac{k}{\theta}\right)} Z(k+\alpha \theta)$.

For the second term which is a nonlinear function in Eq. (1), we consider approximate by the series

$$
f(y)=\sum_{n=0}^{\infty} A_{n}
$$

where the Adomian polynomials $A_{n}$ are calculated by

$$
A_{n}=\frac{1}{n !}\left[\frac{d^{n}}{d \eta^{n}}\left[f\left(\sum_{i=0}^{\infty} \eta^{i} y_{i}\right)\right]\right]_{\eta=0}, \quad n=0,1, \mathrm{~K}
$$

For the differential transform of the fractional Bratu-type differential equation having the form $D^{\alpha} y=f(y)$ where $f(y)$ represents a nonlinear function, the recurrence scheme is given [6] by

$$
\frac{\Gamma\left(\alpha+1+\frac{k}{\theta}\right)}{\Gamma\left(1+\frac{k}{\theta}\right)} Y(k+\alpha \theta)=A_{k}^{\circ}
$$

where $A_{k}^{\circ}$ is obtained from the Adomian polynomials of $f(y)$.

\section{Numerical Results}

\section{Example 1}

We introduce the following Bratu-type differential equation of fractional order [2]

$$
\begin{aligned}
& D^{\alpha} y(x)-2 e^{y(x)}=0, \quad 1<\alpha \leq 2, \quad 0<x<1 \\
& y(0)=0, y^{\prime}(0)=0
\end{aligned}
$$


The exact solution is given by $y(x)=-2 \ln (\cos x)$ for $\alpha=2$. For approximate solution, we apply the fractional differential transform method. Then, the differential transform of $\mathrm{D}^{\alpha} y(x)$ is obtained as

$$
\mathrm{D}^{\alpha} y(x)=\frac{\Gamma(\alpha(k+1)+1)}{\Gamma(1+\alpha k)} \mathrm{Y}(k+1)
$$

where $\theta$ equals to $1 / \alpha$. Since $e^{y(x)}$ is the nonlinear term in Eq. (10), we take advantage of the Adomian polynomials. Then, the Eq. (10) become

$$
\frac{\Gamma(\alpha(k+1)+1)}{\Gamma(1+\alpha k)} \mathrm{Y}(k+1)=2 \%, \quad k=0,1,2, \mathrm{~K}
$$

where $A_{k}$ is the differential transforms of the Adomian polynomials for the exponential nonlinearity $e^{y}$ such that

$$
\begin{array}{ll}
\mathrm{A}_{0}=e^{y_{0}} & \AA_{0}^{\circ}=e^{Y(0)} \\
A_{1}=y_{1} e^{y_{0}} & \AA_{1}=Y(1) e^{Y(0)} \\
A_{2}=\left[y_{2}+\frac{y_{1}^{2}}{2 !}\right] e^{y_{0}} & \AA_{2}=\left[Y(2)+\frac{Y(1)^{2}}{2 !}\right] e^{Y(0)} \\
A_{3}=\left[y_{3}+y_{1} y_{2}+\frac{y_{1}^{3}}{3 !}\right] e^{y_{0}} & A_{3}=\left[Y(3)+Y(1) Y(2)+\frac{(Y(1))^{3}}{3 !}\right] e^{Y(0)} \\
\mathrm{M} & \mathrm{M}
\end{array}
$$

Applying the differential transform to the initial condition, $Y(0)=0$ is obtained for $y(0)=0$. Other differential transform components is found as:

$$
\begin{gathered}
Y(1)=\frac{2}{\Gamma(\alpha+1)} \\
Y(2)=\frac{\Gamma(1+\alpha)}{\Gamma(2 \alpha+1)}\left[2 \frac{2}{\Gamma(\alpha+1)}\right]=\frac{4}{\Gamma(2 \alpha+1)} \\
Y(3)=\frac{\Gamma(2 \alpha+1)}{\Gamma(3 \alpha+1)}\left[2\left(\frac{4}{\Gamma(2 \alpha+1)}+\frac{2}{\Gamma^{2}(\alpha+1)}\right)\right]=\frac{4\left[2 \Gamma^{2}(\alpha+1)+\Gamma(2 \alpha+1)\right]}{\Gamma(3 \alpha+1) \Gamma^{2}(\alpha+1)}
\end{gathered}
$$

Considering the inverse differential transform, the series solution is found as

$$
y(x)=\frac{2}{\Gamma(\alpha+1)} x^{\alpha}+\frac{4}{\Gamma(2 \alpha+1)} x^{2 \alpha}+\frac{4\left[2 \Gamma^{2}(\alpha+1)+\Gamma(2 \alpha+1)\right]}{\Gamma(3 \alpha+1) \Gamma^{2}(\alpha+1)} x^{3 \alpha}+\ldots
$$

The comparisons of the exact solution for $\alpha=2$ and the approximate solutions for the various values of $\alpha$ are demonstrated in Table 1 and Fig. 1. 
Table 1. Comparison of the numerical solutions for the some values $\alpha$ for Example 1 (for $x=0.1$ and $N=n=5$ ).

\begin{tabular}{|l|l|l|}
\hline$\alpha$ & DTM & RKM \\
\hline 1.9 & $1.3814 \mathrm{E}-2$ & $1.3082 \mathrm{E}-2$ \\
\hline 1.8 & $1.8983 \mathrm{E}-2$ & $1.7221 \mathrm{E}-2$ \\
\hline 1.7 & $2.5993 \mathrm{E}-2$ & $2.2504 \mathrm{E}-2$ \\
\hline 1.6 & $3.5472 \mathrm{E}-2$ & $2.9221 \mathrm{E}-2$ \\
\hline 1.5 & $4.8260 \mathrm{E}-2$ & $3.7921 \mathrm{E}-2$ \\
\hline
\end{tabular}

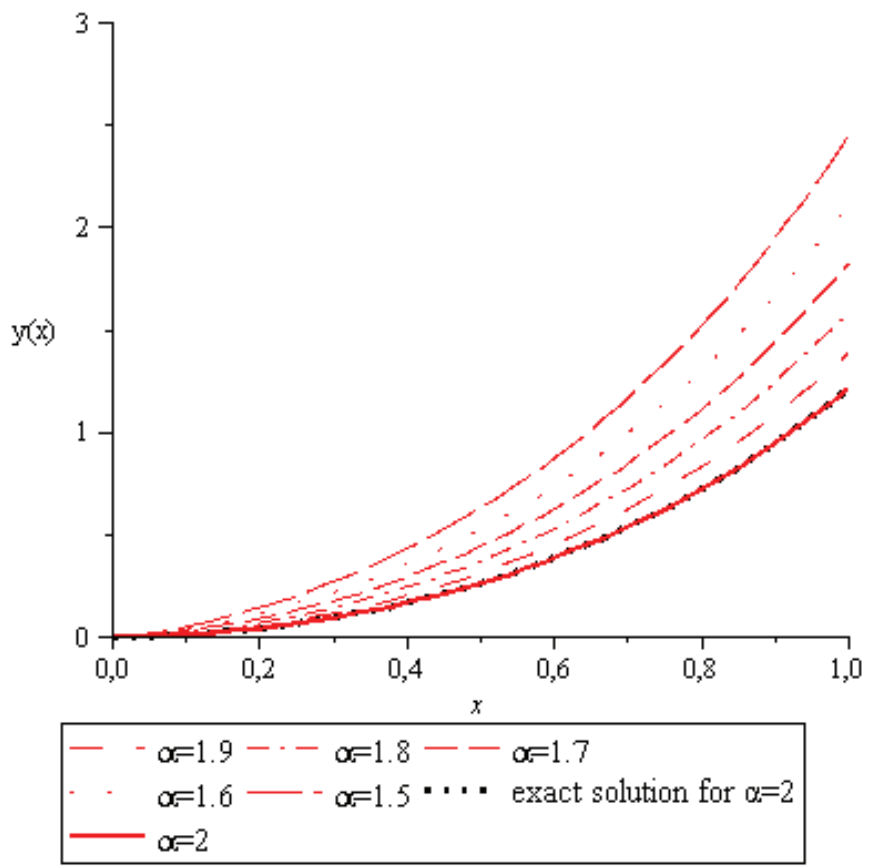

Fig. 1. Comparison the exact solution for $\alpha=2$ with the approximate solutions for the different values $\alpha$ for Example 1.

\section{Example 2}

$$
\begin{gathered}
D^{2 \alpha} y(x)-\pi^{2} e^{y(x)}=0, \quad 0<\alpha \leq 1, \quad 0<x<1 \\
y(0)=0, y^{\alpha}(0)=\pi
\end{gathered}
$$


For $\alpha=1$, the exact solution is $y(x)=-\ln (1-\sin (\pi x))$ [1]. Taking the fractional differential transform and choosing $\theta=1 / \alpha$ yields the recurrence scheme

$$
\mathrm{Y}(k+1)=\frac{\Gamma(1+\alpha k)}{\Gamma(\alpha(k+1)+1)}\left[\pi^{2} \mathscr{A}_{k-1}^{\circ}\right], \mathrm{k}=1,2,3, \ldots
$$

where $\mathcal{A}_{k-1}^{\circ}$ is calculated from of the Adomian polynomials for the nonlinear term $e^{y}$ as

$$
\begin{aligned}
& \AA_{0}^{\circ}=e^{Y(0)} \\
& \mathscr{A}_{1}^{\mathrm{O}}=Y(1) e^{Y(0)} \\
& \mathscr{A}_{2}^{\mathrm{o}}=\left[Y(2)+\frac{Y(1)^{2}}{2 !}\right] e^{Y(0)}
\end{aligned}
$$

M

Applying the differential transform to the initial condition yields $Y(0)=0$. Considering the Maclaurin series, we can choose the differential transform of a trial function corresponding to $y_{1}(x)$ as

$$
Y(1)=\frac{\pi}{\Gamma(\alpha+1)}
$$

Thus, the differential transform components are obtained as

$$
\begin{aligned}
& Y(2)=\frac{\Gamma(1+\alpha)}{\Gamma(2 \alpha+1)} \pi^{2} \\
& Y(3)=\frac{\pi^{3}}{\Gamma(\alpha+1)} \frac{\Gamma(2 \alpha+1)}{\Gamma(3 \alpha+1)}
\end{aligned}
$$

Applying inverse the fractional differential transformation yields the series solution as

$$
y(x)=\frac{\pi}{\Gamma(\alpha+1)} x^{\alpha}+\frac{\Gamma(\alpha+1) \pi^{2}}{\Gamma(2 \alpha+1)} x^{2 \alpha}+\frac{\pi^{3}}{\Gamma(\alpha+1)} \frac{\Gamma(2 \alpha+1)}{\Gamma(3 \alpha+1)} x^{3 \alpha}+\mathrm{K}
$$

Comparing the exact solution for $\alpha=1$ with the approximate solutions for the various values of $\alpha$ are shown in Fig. 2. 


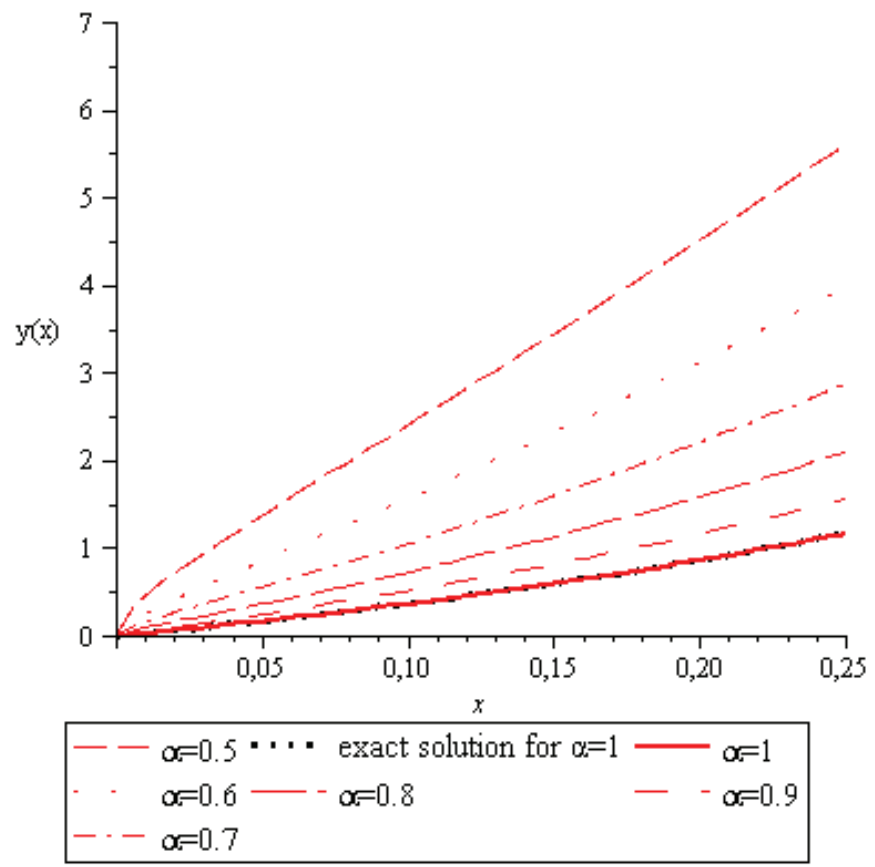

Fig. 2. Comparison the exact solution for $\alpha=1$ with the approximate solutions for the different values $\alpha$ for Example 2.

\section{Example 3}

Consider the initial value problem [1]

$$
\begin{gathered}
D^{2 \alpha} y(x)+\pi^{2} e^{-y(x)}=0, \quad 0<\alpha \leq 1, \quad 0<x<1 \\
y(0)=0, y^{\alpha}(0)=\pi
\end{gathered}
$$

For $\alpha=1$, the exact solution is $y(x)=\ln (1-\sin (\pi x))$. Taking the fractional differential transform and choosing $\theta=1 / \alpha$, then, we obtain the recurrence scheme as follows ;

$$
\mathrm{Y}(k+1)=\frac{\Gamma(1+\alpha k)}{\Gamma(\alpha(k+1)+1)}\left[-\pi^{2} \stackrel{\%}{\mathcal{A}_{k-1}^{\circ}}\right], \mathrm{k}=1,2,3, \ldots
$$

where $\mathcal{A}_{k-1}^{\circ}$ is calculated from of the Adomian polynomials for the nonlinear term, $e^{-y}$. Then, the approximate solution of Eq. (25) by DTM is

$$
y(x)=\frac{\pi}{\Gamma(\alpha+1)} x^{\alpha}-\frac{\Gamma(\alpha+1) \pi^{2}}{\Gamma(2 \alpha+1)} x^{2 \alpha}+\frac{\pi^{3}}{\Gamma(\alpha+1)} \frac{\Gamma(2 \alpha+1)}{\Gamma(3 \alpha+1)} x^{3 \alpha}-\mathrm{K}
$$




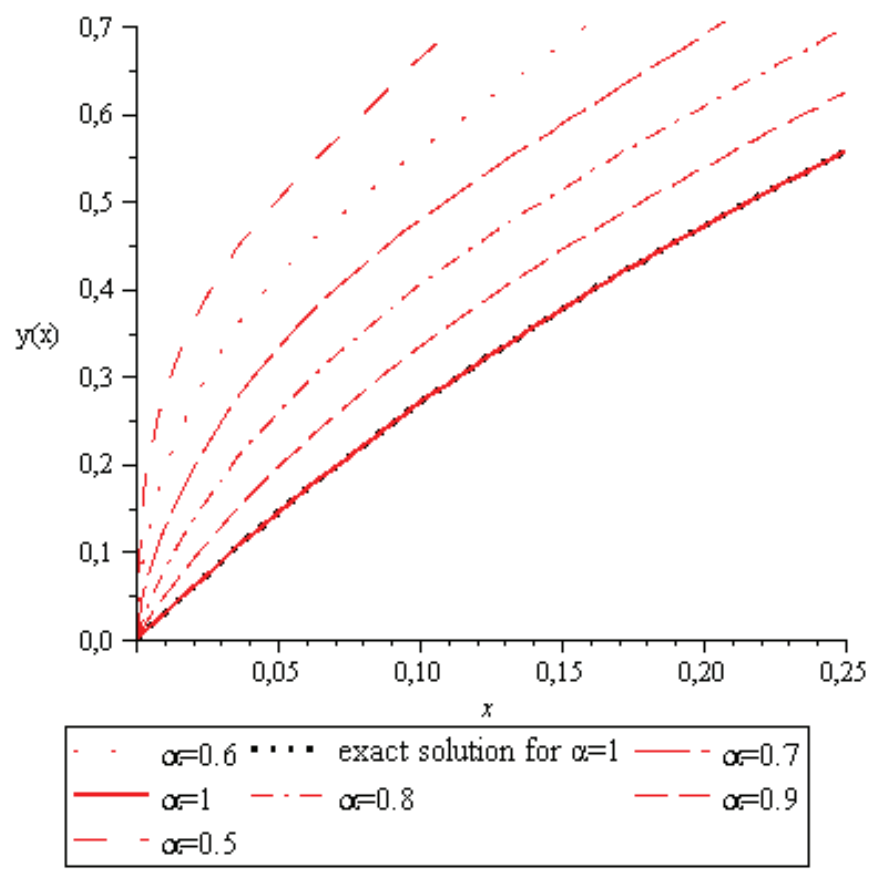

Fig. 3. Comparison the exact solution for $\alpha=1$ with the approximate solutions for the different values $\alpha$ for Example 3.

\section{Example 4}

Consider the following problem [2]

$$
\begin{aligned}
& D^{\alpha} y(x)-e^{2 y(x)}=0, \quad 1<\alpha \leq 2, \quad 0<x<1 \\
& y(0)=0, y^{\prime}(0)=0
\end{aligned}
$$

The exact solution is $y(x)=\ln (\sec (x))$ for $\alpha=2$. The approximate solution is found as

$$
y(x)=\frac{1}{\Gamma(\alpha+1)} x^{\alpha}+\frac{2}{\Gamma(2 \alpha+1)} x^{2 \alpha}+\frac{4 \Gamma^{2}(\alpha+1)+2 \Gamma(2 \alpha+1)}{\Gamma(3 \alpha+1) \Gamma^{2}(\alpha+1)} x^{3 \alpha}+\ldots
$$

The numerical results obtained for $\alpha=1.5(0.1) 1.9$ are compared with the results obtained from the RKM in Table 2. In the Table 3, we present absolute errrors between the exact and approximate solutions for $\alpha=2$. 


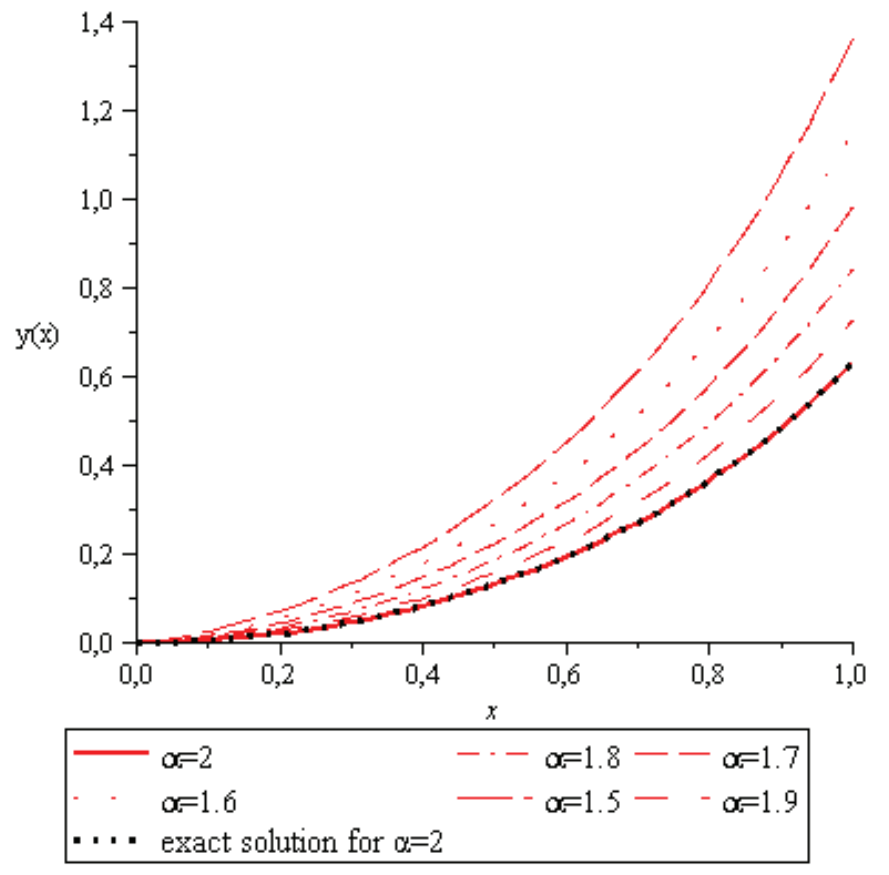

Fig. 4. Comparison the exact solution for $\alpha=2$ with the approximate solutions for the various values $\alpha$ for Example 4.

Table 2. Comparison of the approximate solutions for the various values, $\alpha$ for Example 4 (for $x=0.1$ and $N=n=5$ ).

\begin{tabular}{|l|l|l|}
\hline$\alpha$ & DTM & RKM \\
\hline 1.9 & $6.8982 \mathrm{E}-3$ & $6.8894 \mathrm{E}-3$ \\
\hline 1.8 & $9.4725 \mathrm{E}-3$ & $9.4537 \mathrm{E}-3$ \\
\hline 1.7 & $1.2956 \mathrm{E}-2$ & $1.2917 \mathrm{E}-2$ \\
\hline 1.6 & $1.7652 \mathrm{E}-2$ & $1.7571 \mathrm{E}-2$ \\
\hline 1.5 & $2.3957 \mathrm{E}-2$ & $2.3791 \mathrm{E}-2$ \\
\hline
\end{tabular}


Table 3. Absolute errors for $\alpha=2, N=n=5$.

\begin{tabular}{|l|l|l|}
\hline$x$ & Example 1 & Example 2 \\
\hline 0.1 & $4.4369 \mathrm{E}-8$ & $4.183 \mathrm{E}-6$ \\
\hline 0.2 & $2.2809 \mathrm{E}-6$ & $6.775 \mathrm{E}-5$ \\
\hline 0.3 & $3.1482 \mathrm{E}-5$ & $3.501 \mathrm{E}-4$ \\
\hline 0.4 & $1.7273 \mathrm{E}-4$ & $1.139 \mathrm{E}-3$ \\
\hline 0.5 & $6.3707 \mathrm{E}-4$ & $2.893 \mathrm{E}-3$ \\
\hline 0.6 & $1.8167 \mathrm{E}-3$ & $6.305 \mathrm{E}-3$ \\
\hline 0.7 & $4.3028 \mathrm{E}-3$ & $1.242 \mathrm{E}-2$ \\
\hline 0.8 & $8.7868 \mathrm{E}-3$ & $2.286 \mathrm{E}-2$ \\
\hline
\end{tabular}

\section{Conclusion}

We use the differential transform method for the approximate solutions of fractional Bratutype differential equations. For calculating the differential transform of the exponential nonlinearity, its Adomian polynomials are used in recurrence schemes. It is seen that the differential transform method is implemented simply and effectively to fractional nonlinear differential equations. It is concluded that the present method gives good results.

\section{References}

1. B. Ghazanfari, A. Sepahvandzadeh, J. Math. Computer Sci. 8, 236-244 (2014)

2. E. Babolian, S. Javadi, E. Moradi, Math. Appl. Sci. 39, 1548-1557 (2016)

3. B. Ghazanfari, A. Sepahvandzadeh, Journal of Mathematical Modeling, 2, 143-155 (2015)

4. L. Jin, Int. J.Contemp. Math. Sciences, 5, 153-158 (2010)

5. N. Bildik, A. Konuralp, F. O. Bek, S. Küçükarslan, Appl. Math. Comput. 172, 551-567 (2006)

6. A.Elsaid, Appl. Math. Comput. 218, 6899-6911 (2012)

7. Z. Odibat, S. Momani, V. S. Erturk, Appl. Math. Comput. 197, 467-477 (2008)

8. I. Podlubny, Fractional Differential Equations (Academic Press, 1999)

9. G. C. Wu, E. W. M. Lee, Phys. Lett. A, 374, 2506-2509 (2010)

10. H. R. Marasi, N. Sharifi, H. Piri, TWMSJ. App. Eng. Math. 5, 124-131(2015) 